\title{
Application of water-organic emulsions for the recovery of asphalt concrete
}

\author{
Nikolay Shestakov ${ }^{*}$ and Sergei Putilin \\ Moscow State University of Civil Engineering, Yaroslavskoe shosse, 26, Moscow, 129337, Russia
}

\begin{abstract}
Old asphalt utilization by means of its recycling and re-use in road construction is becoming increasingly important. Its technical and economic efficiency is proved and, in some countries, it reaches ten millions of tons per year. Cold recycling based, in particular, on the use of emulsified bituminous materials is more preferable. However, the modern practice of producing emulsified bituminous materials by using expensive tension-active emulsifiers and equipment of emulsion bases, often of foreign production, makes this seemingly progressive method of recycling quite expensive. In this regard, there is a need for the development of alternative activators based on organic substances. The aim is to study the possibility of water-toluene and water-kerosene emulsions creating and using as activators of cold recycling processes of asphaltic concrete road. The objectives of the study are: selection of the emulsion components ratio as well as the effect activators obtained on the strength and hydrophysical properties of recycled asphaltic concrete. In applying standard analysis of concrete based on asphalt granulate, it was found that the obtained emulsion actively interact with a sealing coat of concrete, based on asphalt granulate, and capable to form composites with a compressive strength of 1.7 MPa that allows their use in the lower layers of road coatings.
\end{abstract}

\section{Introduction}

There is a large number of methods for recycling and reusing of materials that can be applied in the renovation of road coatings. The secondary use of old asphalt concrete by its recycling and application in road construction is becoming increasingly important both in Russia and abroad.

Recently, the cold recycling method of asphalt-concrete coatings is the most valuable among such technologies. It is well known that existing asphalt-concrete layers of road coating are materials of great value that can and should be reused. In cases, where the subgrade capacity of road topping is kept or does not meet the normative value of less than 1.5-2 times, it is possible to carry out an over-haul using the asphalt-concrete coating recycling. When carrying out cold asphalt-concrete recycling, the main task is to process the existing asphalt-concrete coating into asphalt-granulate with its further "revitalization" and puddling. [1-5]

\footnotetext{
Corresponding author: SHestakovNI@mgsu.ru
} 


\section{Materials and Methods}

Asphalt-granulate contains bitumen that has residual operating abilities. The most effective and rational way is the activation of sealing coat on concrete, based on asphalt granulate, by active components. By the nature of the contacted materials, it is most optimal to choose organic solvents as activators, which at low temperatures activate bitumen to the working condition.

It is known that non-polar solvents are: propane, benzene and all other hydrocarbon solvents. Toluene should also be attributed to a group of non-polar solvents, since its small dipole moment does not play a vital role. However, most of the components in bitumen are non-polar to the exclusion of some of the aromatic molecules that have a weakly expressed polarity [6-18]. In this regard, it can be assumed that a dispersion effect occurs when the activators are introduced that causes the interaction between non-polar molecules. Dispersion forces arise as a result of the displacement of the electron shells at the moment of molecules approximation that leads to a short-term and multiple polarization of the molecules. With a certain alignment and the presence of short-term polarization, the molecules gravitate to each other. This is the most common and universal kind of forces of intermolecular interaction, leading to the formation of strong bonds within the composites.

In asphalt-granulate-concrete mixes condensed in the cold state, it is expedient to use bitumen diluted with comparatively light hydrocarbon components, which provide a relatively high rate of thickening of the binder. The most organic solvents include such components. Almost all these components are obtained by stripping from petroleum feedstocks, wherefore, they have good dissolving capacity for bitumen. This determines the choice of active components for the further preparation of activators of cold recycling. For toluene, the electrophilic substitution reactions in the aromatic ring and substitution in the methyl group by the radical mechanism are characterized, so it can certainly be considered as effective component in the revitalization of bitumen sealing coats. However, toluene is of low toxicological concern and low flash point $\left(\mathrm{Tf}=4^{\circ} \mathrm{C}\right)$, so the possibility of using kerosene as an alternative component was considered $\left(\mathrm{Tf}=40^{\circ} \mathrm{C}-72^{\circ} \mathrm{C}\right)$.

The selected components, toluene and kerosene, are highly flammable and toxic, therefore, the most rational way of introduction into the asphalt-granulate is the introduction of these components diluted in an aqueous medium. Since toluene and kerosene are polar liquids, it is not possible to obtain a solution. As known, non-polar liquids do not interact with water, but form emulsions under the condition of colloidal dispersion phase and the presence of emulsifiers, which help to stabilize the systems obtained.

\section{Results and Discussion}

To determine the criteria for the optimal selection of asphalt granules, the asphalt granulate was screened through a set of sieves, according to GOST 9128-2009. The optimization of the mixture intended composition for the construction of road coating layers, until recently, has been associated with an increase in the density of the concrete based on asphalt granulate. The content of the optimal amount of emulsion was calculated by determining the maximum density and optimum soil moisture methodology, since the processes of structure formation and creation of the maximum density of grain coating can be compared with the achievement of maximum soil density. The method for determining the optimum density and maximum soil moisture is regulated by GOST 22733-2002 "SOIL. The method for determining the optimum density". The values obtained are shown in Table 1. 
Table 1. Maximum density of the asphalt granulate

\begin{tabular}{|c|c|c|c|}
\hline No & $\begin{array}{c}\text { Amount of water, } \\
\%\end{array}$ & $\begin{array}{c}\text { Density of the } \\
\text { granulate, } \mathrm{g} / \mathrm{sm}^{3}\end{array}$ & $\begin{array}{c}\text { Density of asphalt- } \\
\text { granulate frame, } \mathrm{g} / \mathrm{sm}^{3}\end{array}$ \\
\hline 1 & 2 & 1,90 & 1,86 \\
\hline 2 & 4 & 1,93 & 1,86 \\
\hline 3 & 6 & 1,97 & 1,86 \\
\hline 4 & 8 & 2,08 & 1,93 \\
\hline 5 & 10 & 2,12 & 1,94 \\
\hline 6 & 12 & 2,07 & 1,85 \\
\hline 7 & 14 & 2,01 & 1,76 \\
\hline 8 & 16 & 1,92 & 1,66 \\
\hline
\end{tabular}

Thus, according to the results of the maximum density value, it is established that the optimum amount of water necessary to ensure the complete wetting of the composite, without the formation of surpluses, is observed with a granulate moisture content of $10 \%$. In further studies of the activator-emulsion content, $10 \%$ of the granulate mass is assigned, the variation occurs due to the active ingredient and its percentage rating in the emulsion.

After the emulsion mixing with the material, the aqueous phase evaporates, the bitumen particles merge into a continuous sealing coat spreading over the surface of the stone material and begin to interact actively with the bitumen components, bringing it to the working condition.

To determine the effect of different activators on the structure of the concrete based on asphalt granulate, images of the surface were obtained. The results of the microstructure of the composites obtained on a scanning electron microscope JSM-6510LV JEOL.

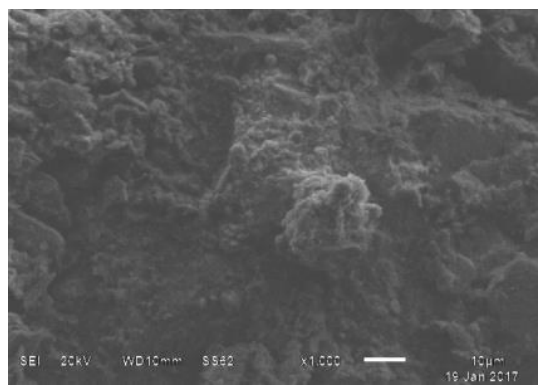

A) Activator based on kerosene, increase $\mathrm{x}$ 1000

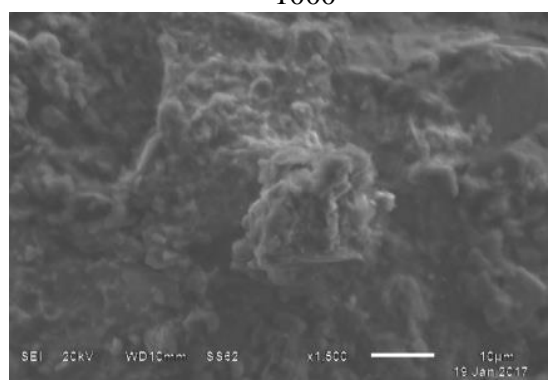

C) Activator based on kerosene, increase $\mathrm{x}$ 1500

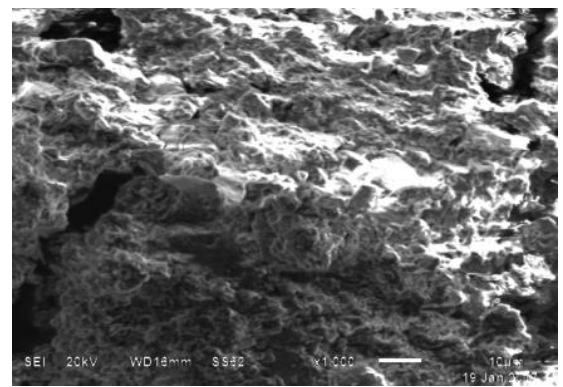

B) Activator based on toluene, increase $\mathrm{x}$ 1000

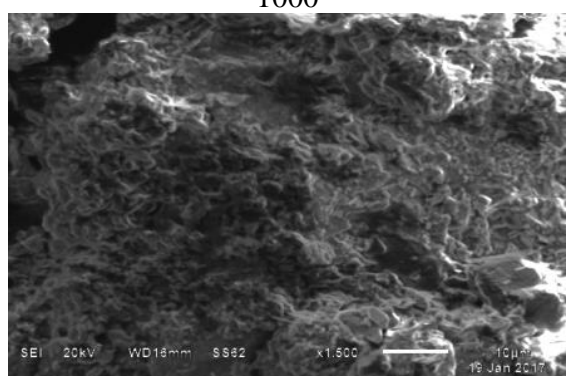

D) Activator based on toluene, increase x 1500

Fig. 1. Microstructure of the resulting composites. 
Using different emulsions, the microstructure of the resulting composites does not have any particular differences. This is due to the same nature of organic components influence on the bitumen sealing coats and the processes of structure formation. The structure of the surface is expressed by smooth monolithic aggregates with a rare presence of cracks in the whole composition. The formation of cracks explains the high index of water saturation and swelling. During the bringing into association of asphalt-granulate with the activator, along with the adsorption processes, a capillary percolation of the cut-back asphaltic road oil components into the crushed grains of the asphalt granulate will be observed. As a result, the viscosity and cohesion of the bitumen sealing coats slightly increases and the strength of the coagulation bond increases as well. During the consolidation of mixture, first of all, physical processes in the bitumen sealing coats occur, leading to its squeezing out from the zones of increased pressure. The free bitumen fills an intergranular space and sealing coat of structured bitumen remains on the grains. In this case, the coagulative bonds become stronger and the strength of the entire system increases. Thus, it can be assumed that the organic activators form thin sealing coats of structured bitumen on the asphaltoganulate grains, which have increased strength. The part of the activator introduced into bitumen would be spent on diffusion processes - the plasticization of old bitumen and filtration into the pores of mineral grains.

The economic efficiency confirmation of the developed technology application was carried out in comparison with a coarse-grained porous asphalt concrete, as with the most frequently used version of the construct.

The calculation is presented for a $1,0 \mathrm{~km}$ long section for a motor-road of III category. The upper layer of the road coating in the calculations is represented by SMA 20 layer of $0.04 \mathrm{~m}$, the lower layers of the coating are $0.13 \mathrm{~m}$. The summary calculated data are presented in Table 2.

Table 2. Comparative economic indicators of pavement construction options

\begin{tabular}{|c|c|c|c|c|}
\hline No & Component & Scope, $\mathrm{m}^{3}$ & Option I & Option II \\
\cline { 3 - 5 } & $\begin{array}{c}\text { Rubble-mastic asphalt } \\
\text { concrete }\end{array}$ & 280 & 2716560,0 & 2716560,0 \\
\hline 2 & $\begin{array}{l}\text { Hot porous coarse- } \\
\text { grained grade II asphalt } \\
\text { concrete }\end{array}$ & 910 & 7272720,0 & - \\
\hline 3 & $\begin{array}{l}\text { Asphaltic concrete } \\
\text { with activator }\end{array}$ & 910 & $\mathbf{-}$ & 1703520,0 \\
\hline Total: & & $\mathbf{9 9 8 9 2 8 0 , 0}$ & $\mathbf{4 4 2 0 0 8 0 , 0}$ \\
\hline
\end{tabular}

When using the road topping design of option II, there is an economic effect observed $126 \%$ savings on materials. The technical and economic comparison shows that the application of the proposed technology gives a significant economic effect, with a slight upgrade of the production line.

The nature of structure formation processes is presented by the preliminary partial dissolution of the solvate layers of the old bitumen, the reduction of the composite to the working condition and subsequent hardening due to the evaporation of the dispersion medium. The resulting composites have sufficiently high strength characteristics up to 1.7 $\mathrm{MPa}$. However, low resistance to water saturation makes possible to use the obtained materials only in the lower layers of asphalt-concrete coatings. The high residual porosity makes possible to use the material as an armored coating layer, taking into account the presence of a weather-resistant coating layer. The technical and economic analysis of the application of cold recycling activators of asphalt-concrete coatings is performed. 
According to the results of experimental studies, a significant economic effect is observed due to the use of a mobile installation, secondary materials and a reduction in transportation costs.

\section{References}

1. N Gornaev, Science and technology in the road industry 2, 20-22 (2004)

2. Foreign experience of cold regeneration. (Application of the regenerative machine ARC-700). Regeneration of asphalt-concrete coatings. Thematic selection.. Ministry of Transport of the Russian Federation (2003)

3. F Turenko, S Filatov, V Shipitsyn, ONV, 3, 36 (2006)

4. S Aleksikov, A Budrudinova, Bulletin of VSU 10, 7, (2012)

5. N Gornaev, V Nikishin, A Kochetkov, Bulletin of the SSTU 3, 34-36 (2007)

6. Li Yumei, Research on old asphalt and the regeneration agent compatibility test, advanced technologies in manufacturing, engineering and materials, PTS 1-3, 11941199 (2013)

7. W. N. Azahar, B Mastura, Jurnal teknologi 78, 105-109 (2016)

8. Hwang Sunn-Jer, Sheu Chyi, Liu Ying-Wei, (8th International Conference on Road and Airfield Pavement Technology, 3, (2013)

9. D Osborn, M Hassan Marwa, D Heather, Transportation research record 2290, 147153, (2012)

10. V Prokopets, S Filatov, T Ivanova, M Tarasova, L Pomorova, Bashkir Chemical Journal 5, 6 (2005)

11. A Maksimenko, D Makatsaria, E Zezyulina, D Bezdnikov, Bulletin of the BelarusianRussian University 2, 4 (2008)

12. D Chernykh, D Stroev, D Zadorozhny, S Gorelov, Engineering Bulletin of the Don, 4, 27 (2013)

13. A Makonkov, A Kuzmina, M Belozor, Bulletin Cherepovets State University 2, 44-48 (2014)

14. V Verenko, A Makarevich, Bulletin KHNADU 40, 14 (2008)

15. R Gataullina, E Rakhmatullina, Innovative Science 2 ,1 (2017)

16. I Leonovich, N Pozhakh, Science and Technology 4, 13(2010)

17. R Musin, Innovative science 8, 1 (2016)

18. I Trofimov, Bulletin KuzGTU 1, 11 (2009) 